\title{
Variations
}

Variations

Revue internationale de théorie critique

$20 \mid 2017$

Expériences oppositionnelles

\section{Le travail intellectuel comme expérience oppositionnelle}

Théorie critique, industrie culturelle et engagement (de l')intellectuel

\section{Christophe Magis}

\section{(2) OpenEdition}

\section{Journals}

Édition électronique

URL : http://journals.openedition.org/variations/843

DOI : 10.4000/variations. 843

ISSN : 1968-3960

Éditeur

Les amis de Variations

Référence électronique

Christophe Magis, «Le travail intellectuel comme expérience oppositionnelle », Variations [En ligne], 20 | 2017, mis en ligne le 25 avril 2017, consulté le 25 février 2021. URL : http://

journals.openedition.org/variations/843; DOI : https://doi.org/10.4000/variations.843

Ce document a été généré automatiquement le 25 février 2021.

Les ami•e•s de Variations 


\section{Le travail intellectuel comme expérience oppositionnelle}

Théorie critique, industrie culturelle et engagement (de l')intellectuel

\section{Christophe Magis}

Pour Isabelle Garo

«Penser même est déjà un signe de résistance, un effort pour ne plus se laisser tromper. " Max Horkheimer, « L'État autoritaire » in Théorie critique.

1 Les mouvements de contestation des dernières années ont contribué à reposer la question ancienne de la place des intellectuels dans les mobilisations sociales. Les deux dernières décennies ont ainsi été l'occasion de réinterroger le concept même d'intellectuel à travers les différents modes de l'engagement ${ }^{1}$. Parmi de telles réinterrogations, bien rares sont celles s'appuyant sur le cadre théorique de la Théorie critique. En effet, les mouvements de protestation actuels ne se limitent plus à dénoncer les injustices systémiques des sociétés que le capitalisme organise et ne laissent aux politiques institutionnelles le soin d'en prendre acte, mais manifestent une opposition directement politique en expérimentant, par la pratique, de nouveaux modes d'organisation et de représentation vers un autre ordre politique alternatif. Il semble ainsi que les expériences émancipatrices se trouvent irrémédiablement dans les engagements pratiques des acteurs de la critique sociale, lesquels s'appuient de plus en plus sur des pratiques numériques qui sont théorisées et inscrites dans des visées stratégiques fines et denses ${ }^{2}$. En conséquence, les obscures circonvolutions théoriques d'un groupe de philosophes écrivant au milieu du $\mathrm{XX}^{\mathrm{e}}$ siècle semblent bien éloignées des possibilités actuelles des expériences oppositionnelles. D'autant que les derniers textes de Théodor Adorno comme de Max Horkheimer, par exemple, et leurs dernières positions, notamment pour le premier au moment des événements de 1968, ont laissé l'image de penseurs s'étant éloignés de la vie politique ${ }^{3}$ et s'étant notamment coupés de toute visée stratégique et organisationnelle ${ }^{4}$. Et s'il est quelquefois rappelé combien 
Herbert Marcuse, baignant dans les années 1960 dans une autre atmosphère de liberté, dans le cadre universitaire californien ${ }^{5}$, a pu, en s'enthousiasmant davantage des mouvements étudiants et des "minorités combattantes", contribuer «à la libération et à la revitalisation de la Théorie critique sous sa forme initiale, celle des années 1930-1940 où l'action et l'organisation politiques n'avaient pas encore été mises horssujet $»^{6}$, il n'en demeure pas moins que l'ultime version du marxisme qu'il a proposé à la fin de sa vie semble pour d'aucuns s'être repliée en une théorie esthétique ${ }^{7}$ butant toujours et toujours sur la question de l'organisation'. À côté de ces limites, les mouvements actuels et les théories qu'ils font émerger paraissent bien plus fluides quant aux catégories de praxis et de théorie, et voient souvent la réconciliation entre les deux, voire entre le travail manuel et intellectuel comme étant déjà largement atteinte soit, classiquement, dans la pratique, soit dans un en-dehors de la division du travail.

2 Il nous semble pourtant que les mouvements comme les réflexions théoriques qu'ils produisent directement ou qui se donnent pour objectif de les penser, gagneraient à se reconnecter aux travaux de la Théorie critique. Et si, dans la constellation actuelle, ce socle théorique a pu connaître quelques tentatives de réactualisations notamment pour penser la crise elle-même ${ }^{9}$, il devrait aussi servir à penser la question du travail intellectuel dans la lutte contre le capitalisme et ses diverses manifestations dans la vie sociale quotidienne contemporaine. Il ne s'agit pas, comme c'est parfois le cas dans certaines exégèses, de chercher à tout prix la réutilisation d'un cadre théorique passé, dont on pourrait toujours s'émerveiller, par rapport à tel ou tel point, de sa (fortuite) «troublante actualité ", mais bien plutôt au contraire de rappeler, à partir de ce cadre, combien la célébration trop rapide de la réconciliation entre certaines catégories d'analyse peut représenter un risque. Alors, le présent article propose de poser les jalons d'une réflexion épistémologique sur la catégorie de travail intellectuel à partir de la Théorie critique, qui permettrait tant de réinterroger les mutations contemporaines du capitalisme tardif et de l'industrie culturelle que de questionner les nombreux débats actuels sur la place des intellectuels critiques par rapport aux luttes sociales. Nous reviendrons ainsi sur un certain nombre de textes de la Théorie critique (et notamment de Adorno et Horkheimer) que nous appréhenderons depuis la question du travail intellectuel, nous questionnerons les limites de cette catégorie à partir des positions théoriques et pratiques des philosophes de l'École de Francfort avant de mettre en débat, à partir de ces linéaments, plusieurs propositions actuelles se proposant de penser les mouvements actuels, notamment par le prisme du rôle à attribuer aux intellectuels et au travail intellectuel dans la lutte sociale et politique contre le capitalisme en crise.

\section{Le travail intellectuel comme catégorie : expérience oppositionnelle et domination}

3 Thème fondamental de la critique marxienne, la question de la division du travail est également très présente dans la Théorie critique dont elle traverse les principaux travaux, et ce dès ses débuts ${ }^{10}$. À la suite des propositions de leur collègue Alfred SohnRethel ${ }^{11}$, Adorno et Horkheimer montrent ainsi dans leur ouvrage commun sur la Dialectique de la raison, combien l'histoire de la domination peut être lue comme une histoire de la domination du travail intellectuel sur le travail manuel. Ils proposent alors en ce sens une relecture matérialiste des mythes fondateurs de la culture 
occidentale. Les activités de défense ont été les premières organisatrices des structures sociales de domination :

«Les chants d'Homère et les hymnes du Rig-Véda datent de l'époque de la propriété foncière et des places fortes, où des peuples guerriers établirent leur domination sur la masse des autochtones vaincus [...]. Le dieu le plus puissant parmi les dieux apparut en même temps que ce monde plébéien où le roi, en sa qualité de chef de la noblesse en armes, condamne les vaincus au travail de la terre, tandis que les médecins, devins, artisans et marchands organisent les rapports sociaux. $\gg^{12}$

Mais elles ont été rapidement secondées et, d'une certaine manière, redoublées par la pensée et le travail des idées :

«L'universalité des idées telle que la développe la logique discursive, la domination dans la sphère conceptuelle, s'est instaurée sur la base de la domination du réel. La substitution de l'unité conceptuelle à l'héritage magique, aux anciennes idées diffuses, exprime l'organisation nouvelle de la vie, conçue pour le commandement par ceux qui sont libres. L'individualité qui, en se soumettant au monde, a appris l'ordre et la subordination n'a pas tardé à assimiler la vérité en général à la pensée organisatrice, dont les distinctions sans ambiguïté sont indispensables à cette vérité pour qu'elle puisse exister. $»^{13}$

5 Le travail intellectuel prend historiquement sa source tant dans la suite de la domination des guerriers vainqueurs sur les peuples vaincus qui sépara les individus libres des esclaves, et au sein du groupe des individus libres, ceux qui se dédièrent au travail de la pensée, s'exemptèrent encore davantage des tâches les plus contraignantes du labeur.

«L'intellect humain, qui a des origines biologiques et sociales, n'est pas une entité absolue, isolée et indépendante. C'est seulement en fonction de la division du travail qu'on en est venu à le décrire ainsi, et afin de justifier cette dernière sur la base de la constitution naturelle de l'homme. On opposa les fonctions directrices de la production (commandement, planification, organisation), en tant que pur intellect, aux fonctions manuelles de la production, considérées comme formes basses et impures du travail, comme travail d'esclave. Et ce n'est pas par hasard si ce qu'on appelle la psychologie platonicienne, dans laquelle l'intellect était pour la première fois opposé aux autres "facultés" humaines et plus particulièrement à la vie instinctuelle, fut conçue sur le modèle de la division des pouvoirs dans un État rigidement hiérarchisé. ${ }^{14}$

6 Formellement, ce travail de la pensée rejoint par ailleurs celui de la contemplation et de la création artistique. Il est dans les deux cas privilège de ceux qui n'ont pas eu dans l'histoire à s'adonner aux tâches sociales les plus pénibles de la production. Le passage des sirènes dans l'épopée d'Ulysse est, pour Adorno et Horkheimer, ce point de constitution du sujet moderne comme maître et jouisseur de la contemplation: «Auditeur passif, le ligoté écoute un concert comme le feront plus tard les auditeurs dans la salle de concert, et son ardente imploration s'évanouit déjà comme les applaudissements. C'est ainsi que la jouissance de l'art et le travail manuel se scindent à la fin de l'ère préhistorique. $»^{15}$ Tout comme la philosophie, le plaisir esthétique en tant qu'activité prend racine dans sa séparation des tâches les plus contraignantes du travail manuel et les deux activités portent alors la domination dans leur geste même.

7 On peut s'arrêter un instant sur ce que cette affirmation porte de signification quant à la position des deux philosophes qui la posent. Par exemple, Adorno, philosophe, dont on connaît le goût pour certaines avant-gardes artistiques - notamment pour la nouvelle musique de la seconde école de Vienne - et, a fortiori, pour un ensemble d'éléments feutrés de la culture bourgeoise, appartient complètement à cette catégorie 
sociale à laquelle appartient également Hegel, qu'il critique dans les Trois études qu'il lui consacre : celle de la lignée des privilégiés qui «à la faveur de la séparation entre travail manuel et travail intellectuel, [...] se sont arrogé le travail intellectuel, plus facile malgré toutes leurs protestations ${ }^{16}$. Plusieurs éléments biographiques que les deux penseurs ont pu livrer eux-mêmes de manière fugace au détour de leurs écrits ${ }^{17}$, ou qui ont pu être rapportés par d'autres, laissent ainsi clairement entrevoir leur position de naissance dans les rapports de production. Par exemple, dans un petit texte de 1970, Horkheimer rappelle l'origine de son ami et collaborateur en même temps que la sienne propre: «Nous sommes tous deux d'origine bourgeoise et nous avons aussi appris à connaître le monde par l'intermédiaire de nos pères qui étaient des industriels. [...] Nous avons vécu la Première Guerre mondiale et, par la suite, nous n'avons pas étudié pour faire carrière, mais parce que nous voulions apprendre à comprendre le monde ${ }^{18}$. Ayant tout de même fait carrière, les deux philosophes se retrouvent dans les positions qu'ils critiquent en se proposant d'historiciser l'exercice même du travail philosophique. Ils se risquent en permanence à tomber dans cette position contre laquelle Marx et Engels mettaient déjà en garde en moquant les jeunes hégéliens (et notamment leurs collègues Bauer, Feuerbach et Stirner) qui avaient fini par justifier leur propre séparation de la pratique en posant philosophiquement la séparation définitive entre formes de conscience et être social. Les auteurs de l'Idéologie allemande mettent ainsi bien en rapport cette séparation, telle qu'elle apparaissait alors aux philosophes, avec la propre situation sociale de ces derniers dans la division entre travail manuel et intellectuel, qui rend effective la division du travail :

« À partir de ce moment, la conscience peut vraiment s'imaginer qu'elle est autre chose que la conscience de la pratique existante, qu'elle représente réellement quelque chose sans représenter quelque chose de réel. À partir de ce moment, la conscience est en état de s'émanciper du monde et de passer à la formation de la théorie "pure", théologie, philosophie, morale, etc. ${ }^{19}$

8 Les philosophes de la Théorie critique ont bien conscience de ce risque, qu'ils critiquent également. Mais ils notent dialectiquement que cet ancrage dans la domination est aussi ce qui a permis la constitution du sujet transcendantal et de l'esprit. Le geste philosophique comme celui de la contemplation esthétique, quoiqu'étant le privilège de ceux qui n'ont pas à travailler à d'autres tâches plus difficiles pour leur propre subsistance, devraient tout de même être un privilège dirigé vers sa propre abolition en tant que privilège. Dans l'activité de la conceptualisation par exemple, le travail intellectuel nie la difficulté du travail manuel concret et provoque la nécessité absolue d'une réconciliation en poussant au bout sa contradiction avec le travail manuel, tout en planifiant les conditions de la réconciliation. La méditation transcendantale du " pur » esprit, dans l'activité du théoricien, n'est rien d'autre que la tentative de l'individu de s'échapper de la lourdeur la plus asservissante du travail manuel, posant par-là ce dernier comme son autre dans une synthèse abstraite ${ }^{20}$. La théorie "se réfléchit elle-même et ce faisant, s'abroge comme pure théorie $»^{21}$. De son côté, l'œuvre d'art, qui jouit également d'une autonomie relative par rapport au social ${ }^{22}$ liée justement en partie à cette position historique de marginalité dont l'artiste bénéficie qui n'a pas, comme travailleur intellectuel, à s'occuper de tâches plus directement productives - que ce soit dans l'Histoire grâce au mécénat ou à sa position d'héritier -, doit également faire vivre et mettre en avant en permanence la tension entre l'impulsion individuelle spontanée et la structure sociale, laisser entrevoir, au niveau du sensible, l'image de ce que pourrait être une réconciliation. À travers la pensée 
dialectique et sa catégorie fondamentale de négation (qui est autant centrale pour le travail philosophique que pour la production artistique, tels que l'entendaient les philosophes de la Théorie critique ${ }^{23}$ ) le travail intellectuel doit se critiquer lui-même, portant alors la possibilité de l'émancipation collective dans une synthèse à construire de sa contradiction avec le travail manuel.

Bien sûr, à ce moment de l'argumentation, il pourrait être rétorqué que c'est justement ici que les propositions de Marx doivent être prises en compte. Dans la perspective marxienne, c'est bien dans la pratique seule et notamment la pratique politique et révolutionnaire - qui ne peut se limiter à la simple pratique de l'artiste ou du théoricien et au seul niveau des formes esthétiques ou philosophiques - que ce dépassement peut se faire. Mais il faut toutefois également prendre ici en compte l'élément majeur qui accompagne la réflexion de la Théorie critique et qui tempère cette possibilité : la problématique de l'industrie culturelle. Avant de présenter cette problématique, notons ici un premier élément d'après ce qui vient d'être dit. En tant que catégorie, le travail intellectuel est par définition le lieu d'une expérience oppositionnelle. En effet, comme nous venons de le voir, l'histoire de la catégorie est pour Adorno et Horkheimer une histoire de son opposition à d'autres types de labeurs, plus aliénants, desquels les dominants se sont séparés. Ainsi entendue, la catégorie de travail intellectuel contient en réalité l'expérience oppositionnelle en même temps que la domination qui l'a fait naître : on pourrait dire qu'opposition et domination sont ici les deux faces de la même médaille. Le travail intellectuel s'oppose, comme travail, aux activités les plus directement asservissantes desquelles il s'émancipe, témoignant ainsi de la domination historique des individus qui le pratiquent sur ceux qui en sont maintenus à l'écart. Il convient de garder à l'idée cette définition de la catégorie de travail intellectuel dans l'opposition aux forces les plus aliénantes du social plutôt que de chercher de manière plus positive à le caractériser par rapport à la mise en œuvre effective de processus cognitifs dans le travail. Aujourd'hui, cette distinction nous paraît importante à l'heure où les développements du capitalisme invitent à parler de capitalisme cognitif ou à qualifier la période actuelle d'ère "post-industrielle " pour justement décrire les activités de travail routinier qui, pour autant, s'appuient sur une intégration accrue des capacités intellectuelles. L'impression conséquente qu'une grande partie du travail actuel très exploité et aliéné relève pourtant de formes de travail intellectuel dans les formes nouvelles d'exploitation qui fleurissent notamment autour des plateformes numériques ${ }^{24}$ ne doit ainsi pas laisser croire qu'une catégorie de «travail intellectuel» comme celle que nous venons de définir d'après la Théorie critique est désormais caduque. Au contraire, et comme nous allons le voir, une telle catégorie permet de critiquer les conséquences de l'industrie culturelle.

\section{Kulturindustrie : vers la fin de l'opposition travail intellectuel/travail manuel?}

10 La société industrielle, dont Adorno et Horkheimer proposent de retracer l'avènement dans leur célèbre essai sur La dialectique de la Raison, est bien un prolongement de la domination historique de la tête sur la main, de cette domination sociale du savoir et de ceux qui concourent à le produire et surtout à l'utiliser :

«Le savoir, qui est un pouvoir, ne connait de limites ni dans l'esclavage auquel la créature est réduite, ni dans la complaisance à l'égard des maîtres de ce monde. De 
même qu'il sert tous les objectifs de l'économie bourgeoise à l'usine et sur le champ de bataille, il est aux ordres de ceux qui entreprennent quelque chose, quelles que soient leurs origines. ${ }^{25}$

Mais pour les besoins du pouvoir, justement, le savoir ne peut se risquer à contenir la possibilité du moindre dépassement. C'est bien là, à notre sens, un élément fondamental qu'il convient de retirer du chapitre central de l'ouvrage et de son concept célèbre d'industrie culturelle - bien que le choix de traduction française de Kulturindustrie par «La production industrielle de biens culturels » invite plutôt à le considérer comme une analyse socio-politique et économique de la sphère industrielle de production et de la mise en marché des biens symboliques ${ }^{26}$. Ce qui nous semble en jeu dans ce chapitre c'est, plus fondamentalement et en filigrane, une analyse de la possibilité d'un travail intellectuel véritable (i.e. : fidèle à son concept, cf. supra) dans la société du capitalisme avancé. À un âge industriel où la logique de la marchandise a conquis l'essentiel des activités de la vie sociale, l'industrie culturelle décrit, en tant que concept, ce mouvement qui réduit l'ambition du travail de l'esprit à la trivialité du toujours-identique au point que, offert contre monnaie sonnante et trébuchante comme divertissement culturel ou comme higher education, il n'échappe jamais à l'individualité du travail manuel, rendant ridicule sa prétention à être autre chose.

D'un côté, il s'abîme en babillage pseudo-artistique. Les fameux travaux d'Adorno "sur la musique populaire $»^{27}$, le $« \mathrm{jazz} »^{28}$ ou la musique de cinéma ${ }^{29}$ analysent à chaque fois cette situation de manière spécifique à partir de matériaux différents pour ce qui concerne le musical. Ils montrent comment la nécessité de succès, dictée par la logique de la rentabilité économique, a atteint jusqu'à la structure même des œuvres. Les effets, par exemple, ou ce qu'Adorno appelle les « instants de charme» (telle texture sonore de l'orchestration, tel motif musical envoutant, tel mouvement harmonique inattendu, telle figure rythmique exaltante) ont ainsi acquis, dans ces "œuvres", une place absolument déterminante. Le propos n'est évidemment pas d'arguer pour la nécessité d'une esthétique forcément ascétique (comme ça a quelquefois pu être reproché au philosophe), mais de remarquer que la fonction de ces moments de charme s'est transformée et, avec elle, la possibilité d'une esthétique considérant les pièces musicales comme totalité. Ces «instants de charme » avaient en effet une fonction particulière dans des œuvres d'art pré-industrie culturelle et comprises chacune comme un tout: celle d'une révolte contre la loi formelle, la rationalité et les conventions que l'œuvre incorporait. Leur exploitation au service de la rentabilité et donc du succès, leur fait perdre cette force que leur conférait auparavant leur rapport critique à la structure d'ensemble. Ils sont, dans l'industrie culturelle, exploités pour eux-mêmes et deviennent alors des éléments de coloriage, susceptibles de maquiller les structures de la musique industrielle qui, dans le même temps et pour des raisons de nécessité de reproduction de ce qui a marché, se sont sclérosées: "au service du succès, ils renoncent eux-mêmes au caractère d'insoumission qui leur était propre. [...] Dans l'isolement, les charmes perdent leur puissance et finissent par constituer des poncifs $\Perp^{30}$. Dès lors, bien que travail intellectuel, la composition - savante comme populaire d'ailleurs ${ }^{31}$ - et, au-delà, la production des œuvres d'art comme activité, n'est plus le lieu de cette possible critique et réconciliation, dans la forme esthétique, des tensions qui traversent le social. Il devient un travail qui n'a plus d'intellectuel que la fonction sociale de son producteur (qui ne sauve ainsi que sa propre pratique de la pénibilité du travail manuel), mais porte à tous les niveaux les caractères d'aliénation et d'individualité du travail manuel, avec lequel il n'est plus en opposition dans 
l'expérience du travail. Il reste simplement en opposition avec lui au niveau de la domination sociale du producteur de culture, qui reste un "col blanc», sur le producteur non-culturel.

13 De l'autre côté, le travail intellectuel devient simple huilage des rouages de la machinerie industrielle. Alors que le secteur de la production théorique a été conquis par la logique du capital et sa raison instrumentale - faisant au passage de l'éducation un lieu stratégique de création de profit et de contrôle - l'activité philosophique et scientifique s'est réduite à n'être plus soit, qu'un bavardage de salon pour ceux qui peuvent, en choisissant de faire des études longues plutôt que de s'engager dans des activités plus immédiatement rémunératrices, goûter par là leur privilège de classe soit, qu'une formation à l'indentification et l'application de procédures techniques dans la planification des tâches de l'appareil de production. Le triomphe de la pensée positive et son "intelligence pragmatique» dans l'activité de la philosophie et des sciences sociales est la matérialisation de ce mouvement de l'industrie culturelle dans la pensée. Dans un essai rassemblant une série de cours que Horkheimer a donné au moment de la rédaction à quatre mains de la Dialectique de la raison, le philosophe formule ainsi cette situation :

«[La liberté de contemplation] fut toujours le privilège de certains groupes qui, automatiquement, construisaient une idéologie, hypostasiant leur privilège en vertu humaine. Ainsi cette liberté servait des desseins idéologiques bien réels, en glorifiant les exemptés du travail manuel. Et de là vient la méfiance qu'éveille ce groupe. En fait l'intellectuel de notre ère n'est pas sans subir des pressions : l'économie les exerce sur lui pour satisfaire les exigences toujours changeantes de la réalité. Par conséquent la médiation tournée vers l'éternité est remplacée par l'intelligence pragmatique, tournée vers le moment qui vient. Au lieu de perdre son caractère de privilège, la pensée spéculative est totalement liquidée. $\|^{32}$

En se fonctionnalisant, la pensée ne devient qu'une vague redite de l'appareil de production: "Toute phrase qui n'est pas équivalente à une opération de cet appareil semble aussi dépourvue de sens pour le profane que pour le sémanticien contemporain, lequel donne à entendre que seule la phrase purement symbolique, purement opérationnelle, c'est-à-dire la phrase sans aucun sens, a un sens. $»^{33}$. Les travaux de Herbert Marcuse sur la société unidimensionnelle, par exemple, sont une analyse de cette réduction du travail intellectuel à la pensée positive ${ }^{34}$. Également, les attaques célèbres d'Adorno à l'encontre du positivisme dans les sciences sociales ${ }^{35}$ sont à comprendre comme une critique de ce mouvement de colonisation de la production théorique par la logique du capital et sa rationalité instrumentale. Personnifié, à l'heure de l'industrie culturelle, dans les activités du consultant, de l'expert et du manager, le travail intellectuel a été simplement vidé de son intérêt social, qui avait pu justifier - quoiqu'abusivement - la libération des activités de production théorique du labeur physique. Il ne sert désormais plus qu'à enregistrer et viser la reproduction de ce qui est là et, par là même, la reproduction du capital comme de la société que ce dernier administre, en général par l'utilisation d'une logique mathématique dispensant de l'exercice même de la pensée :

«Des opérations logiques compliquées sont exécutées sans que soient effectivement accomplis tous les actes intellectuels sur lesquels les symboles mathématiques et logiques sont basés. Une telle mécanisation est, de fait, essentielle à l'expansion de l'industrie. Mais si elle devient le trait caractéristique des esprits, si la raison même est instrumentalisée, elle assume une sorte de matérialité aveugle, devient un fétiche, une entité magique, acceptée plutôt qu'expérimentée sur le plan intellectuel. ${ }^{36}$ 
Cette logique mathématique, grandement révérée plutôt que questionnée par ce que devrait être une production scientifique à la hauteur de la catégorie du travail intellectuel dans laquelle elle s'insère, finit par fermer la société dans l'univers clos d'un langage scientifique vidé de toute expérience. Ainsi, commentant la critique adornienne de l'usage non-réfléchi des statistiques en sciences sociales, Alexander Neumann montre combien cette méthodologie est interprétée comme la «méthode juste " d'une "société fausse ", tendant à présenter comme caduque la nécessité d'un travail intellectuel qui, s'opposant tant à l'aliénation du labeur manuel qu'à la réification de la vie académique par le mouvement de l'industrie culturelle, pourrait contenir l'expérience de ce que serait une pensée autre : «Les statistiques contribuent [...] à une définition exacte de la société comme fait accompli, mais ne font en aucun cas apparaître les potentialités subjectives de son dépassement ${ }^{37}$.

Ici encore et comme dans le cas de la production culturelle, le travail de la pensée n'a plus d'intellectuel que la fonction sociale qu'on reconnaît à son producteur, l'industrie culturelle éliminant la possibilité d'un travail intellectuel authentique de production de théorie mais tout en conservant, néanmoins, la domination sociale formelle que ce dernier contient :

«Si c'est par l'industrie culturelle et celle de la conscience, ainsi que par les monopoles de l'opinion, que l'aménagement de la société empêche - que ce soit de manière automatique ou bien planifiée - la connaissance et l'expérience les plus élémentaires des processus les plus menaçants ainsi que celles des idées et des théorèmes critiques essentiels; si, bien au-delà, l'aménagement de la société paralyse la simple capacité de se représenter le monde sous une apparence concrètement autre que celle qu'il a pour ceux dont il se compose - c'est-à-dire comme une force écrasante -, alors l'état fixé et manipulé de l'esprit devient violence réelle, celle de la répression, de même qu'un jour le contraire de cette dernière, l'esprit libre, avait voulu la supprimer. " $^{38}$

Ce retournement du travail intellectuel en son contraire, la dispense de la pensée, qui est consécutif à la conquête des activités de l'esprit par la logique du capital et sa raison instrumentale, est aujourd'hui amplifié, notamment au niveau de la production théorique, encadré par la prégnance de notions comme celles d'«économie de la connaissance »dans les politiques pour l'enseignement supérieur et la recherche et l'injonction subséquente à la production, au sein des universités, de recherches porteuses d'innovation industrielle permettant un accroissement de la compétitivité économique ${ }^{39}$. L'obtention de financements conditionnée à la réponse à des appels d'offres, en fonction d'intérêts tant privés que politico-institutionnels, où les chercheurs sont mis en concurrence, selon une logique bureaucratisée, semble n'attendre des travailleurs intellectuels universitaires qu'une bonne connaissance des termes-clef et des procédures en vigueur dans l'industrie sur lesquelles ils sont invités à modeler tant leur savoir que leur langage ${ }^{40}$. On voit, ici encore, combien en réalité, le travail intellectuel est enjoint à se limiter à n'être qu'une fonction de l'appareil de production. L'expérience oppositionnelle qu'il pouvait représenter se réduit comme peau de chagrin, alors pourtant que la domination qui l'avait fait naître augmente. Il ne devient plus qu'une opposition de personnes et de statuts dans la hiérarchie de la division (internationale ${ }^{41}$ ) du travail. 


\section{Engagement : reposer le caractère oppositionnel du travail intellectuel}

18 Il nous semble, dès lors, que c'est à partir de cette situation, telle qu'elle est analysée par les théoriciens critiques qu'il convient de réfléchir les positions de ces derniers en ce qui concerne notamment les possibilités de l'engagement concernant le travail intellectuel. À ce titre, la pratique comme les textes théoriques d'Adorno apportent quelque éclairage significatif, notamment en ce qui concerne l'engagement du producteur intellectuel, qu'il soit artiste ou théoricien.

Il est peut-être plus aisé, pour comprendre la perspective adornienne - qui rejoint les thèmes principaux d'autres membres de la Théorie critique comme Horkheimer ou Marcuse -, de commencer par l'artiste. Dans un petit texte publié en 1962 d'après une conférence radiophonique, Adorno interroge la conception de l'artiste engagé telle qu'elle est développée par Jean-Paul Sartre dans son manifeste Qu'est-ce que la littérature? Nous n'aurons pas la place ici de rendre un parfait hommage à la finesse des propositions de Sartre; nous nous contenterons, en assumant le biais que ce choix peut induire, de reprendre ici la lecture qu'en fait Adorno et qui nous semble suffire à la présente démonstration. Il s'agit, pour le philosophe francfortois, de dépasser l'opposition sur laquelle s'est stabilisée la réflexion artistique depuis la publication de la proposition sartrienne entre deux alternatives : celle de l'« art pour l'art " (défendue notamment par Paul Valéry), d'un côté, et celle que justement lui oppose Sartre, de l' « art engagé »:

"Chacun des termes de cette alternative se nie lui-même en même temps que l'autre : l'art engagé, parce qu'il supprime la différence entre l'art et la réalité, alors qu'il se distingue nécessairement de celle-ci puisqu'il est de l'art ; et l'art pour l'art, parce qu'en se voulant un absolu il nie aussi cette relation obligée à la réalité implicitement contenue dans son émancipation par rapport au concret, qui est son a priori polémique. Entre ces deux pôles, la tension qui a animé l'art jusqu'à une époque toute récente se défait. $»^{42}$

C'est donc une autre voie qu'Adorno défend, qui interroge autrement les rapports entre forme et contenu. Pour Sartre, l'art engagé concerne par essence l'œuvre littéraire : si, pour lui, « le peintre est muet ", « l'écrivain, au contraire, c'est aux significations qu'il a affaire $»^{43}$. Cette spécificité conceptuelle de l'art littéraire confère à ses auteurs une grande responsabilité - selon une conception finalement proche de celle défendue par Bertolt Brecht dans sa proposition de théâtre épique : l'écrivain doit utiliser le médium à sa disposition pour adresser des messages aux lecteurs susceptibles de les éclairer sur le monde. Cette position qui prête aux écrivains une responsabilité importante dans la prise de conscience, est à la fois très ancrée dans l'histoire de la littérature ${ }^{44}$ et encore aujourd'hui largement partagée, tant par des écrivains que par des théoriciens littéraires, le médium qu'ils manient étant encore souvent considéré comme particulièrement puissant pouvant tantôt contribuer à la mise en accusation des ordres existant ou à leur renforcement ${ }^{45}$.

21 Pour Adorno, l'art ne peut être ainsi réduit à la simple communication sans voir sa propre fonction critique amoindrie ${ }^{46}$. Comme nous le voyions supra, cette dernière réside bien plutôt, pour le philosophe allemand, dans la forme des œuvres (qu'elles soient littéraires ou non), lieu où peut se jouer tant la cristallisation des tensions du social que leur dépassement. L'engagement de l'artiste, pour Adorno mais aussi pour 
d'autres théoriciens critiques comme Marcuse, est donc un engagement du côté de la forme ; c'est par ce travail continu de la forme, dans lequel l'œuvre capte et incorpore mimétiquement des éléments du social et subsume leurs tensions dans un usage éclairé des techniques de rationalisation artistiques pour renvoyer à la société, par le sensible, une «image belle de la libération $»^{47}$, une image de ce que serait une société qui utiliserait la raison à des fins véritables d'émancipation individuelle et collective mouvement qu'Adorno appelle "synthèse non-violente $»^{48}-$, que l'artiste assume le concept véritable de l'art comme travail intellectuel. Dès lors, la conscience politique directe et revendiquée ou non de l'artiste n'importe pas vraiment à cette notion d'engagement. «Plus une œuvre est immédiatement politique, plus elle perd son pouvoir de décentrement et la radicalité, la transcendance de ses objectifs de changement. $»^{49}$ Pour les auteurs de la Théorie critique, et notamment pour Adorno, ce sont bien les artistes des avant-gardes, ayant cherché (ou cherchant) à assumer coûte que coûte ce maintien du travail de la forme quitte à se détourner d'un public, dont une industrie culturelle puissante limite les prétentions en matière artistique aux simples biens qu'elle produit, qui poursuivent la fonction critique du travail intellectuel. Et leurs œuvres, si elles ne parviennent plus, dans leur totalité formelle, à atteindre la réconciliation, sont alors bien plus "parlantes" quant au cours du monde et à la nécessité de le renverser, que les messages des écrivains engagés qui risquent toujours de s'abîmer en pure propagande :

«Leur caractère de nécessité implacable contraint à ce changement de comportement que les œuvres engagées ne font que réclamer. Si l'on a été pris un jour dans la machine de Kafka, on ne pourra plus jamais être en paix avec le monde, ni se contenter de juger que le monde va mal: le moment rassurant que recèle la constatation résignée de la toute-puissance du mal est comme détruit à l'acide. $»^{50}$

22 C'est ainsi par leur comportement spécifique, plutôt que par ce qu'elles " ont à dire » que les œuvres d'art peuvent résister, comme fruit du travail intellectuel et par le sensible, à la logique instrumentale et à sa matérialisation dans l'industrie culturelle. D'ailleurs, face aux reproches récurrents d'intellectualisme faits à la musique d'avantgarde d'Arnold Schœnberg, par exemple, Adorno rappelle qu' " [e]n vérité, Schœnberg fut un artiste naiff ; les théories maladroites par lesquelles il s'efforçait de justifier ses œuvres n'en sont pas la moindre preuve. Nul, sinon lui, obéissait à une vision musicale débordante et spontanée ${ }^{51}$. Il ne convient pas tant, pour les artistes, de se forger une conscience politique que de chercher toujours à maintenir, dans les marges possibles que laisse l'industrie culturelle, l'espace dans lequel leur travail de création, comme travail intellectuel, peut s'opposer aux tendances les plus aliénantes du social, laissant entrevoir au niveau sensible ce que serait un monde autre, où toute impulsion n'aurait pas été déjà domestiquée par l'industrie culturelle.

Cette position, toute convaincante qu'elle puisse sembler, ne parait toutefois pas directement transposable au travail de la théorie. En effet, ce dernier ne peut totalement être assimilé au travail artistique, justement parce que - pour paraphraser Adorno - il s'agit d'un travail de production théorique : le domaine de l'art n'est pas celui de la pensée scientifique. S'il est certain, pour le philosophe allemand, que la séparation de l'art et de la science, au sein du travail intellectuel est aussi le résultat d'un processus historique, celui de « l'objectivation du monde au cours de sa démystification croissante ", il n'en demeure pas moins qu' " on ne saurait faire réapparaître d'un coup de baguette magique une conscience pour laquelle l'intuition et le concept, l'image et le signe seraient une seule et même chose, si tant est qu'elle ait jamais existé, et sa 
reconstitution serait un retour au chaos $\aleph^{52}$. C'est toutefois bien, à nouveau, dans la forme à donner à ce travail théorique, que peut résider l'engagement qui devrait, ici, pouvoir faire corps avec l'engagement du théoricien quant à son sujet d'analyse, son exploration de celui-ci et la visée de sa production théorique. Car, ici encore, la production intellectuelle ne peut être qu'un travail aux prises avec des tendances contradictoires. En effet, il s'agit de déjouer, dans la production de théorie comme travail intellectuel, les tendances aliénantes de la logique instrumentale qui ont fini par changer les sciences sociales et la philosophie en atelier de collecte des faits, étendant le pouvoir que ces derniers ont sur le monde, mais sans pour autant renoncer à la théorie.

Il convient alors, pour Adorno (mais aussi pour d'autres théoriciens critiques), d'assumer dialectiquement cette tension dans le travail de la production théorique et de ne se soumettre pleinement à aucune des logiques, tout en faisant l'expérience de chacune d'elles : elles sont aussi la manifestation des contradictions qui traversent le social. Il faut admettre, tant face à la pensée positiviste qui prétend avoir réduit le monde en formules - niant par-là la nécessité de toute interprétation -, que face à croyance futile en un monde de pures intensités et différences - rendu alors ininterprétable -, que la production théorique, dans le monde irréconcilié, ne peut être qu'une exploration perpétuelle de la tension entre sujet et objet, entre concept et expérience. Dès lors, cette exploration ne peut se contenter de catégories de pensée ou d'analyse qui atténueraient les contradictions en les considérant comme de simples erreurs lors de l'établissement des enquêtes: «Les rapports sont neutralisés sous la forme de notions comme le "pouvoir" ou le "contrôle social". Dans ces catégories de ce genre l'aiguillon disparaît, et par là, serait-on tenté de dire, ce qui, à même la société, constitue à proprement parler le social [das Soziale], sa structure $»^{53}$. Ainsi,

«Celui qui ne se laisse pas barrer l'accès à l'expérience de la primauté de la structure sur les états de choses ne tâchera pas d'emblée - comme le font la plupart du temps ses contradicteurs - de dévaloriser des contradictions en les rapportant à la méthodologie, c'est-à-dire en les qualifiant d'erreurs de raisonnement, ni de les écarter au profit de l'univocité de la systématique scientifique. Au lieu de cela, il poursuivra les contradictions jusque dans la structure, qui a été antagoniste depuis que la société, au sens le plus noble du terme, existe, et qui continue à l'être $[. ..]{ }^{54}$

Il faut également accepter que toute méthodologie, aussi objective qu'elle soit, ne pourra jamais mener en soi à "l'objectivité de l'objet de recherche $»^{55}$ et que cette dernière n'est jamais que médiate, particulière et, finalement, décevante... mais pour autant, absolument nécessaire. Dans un monde où une alliance barbare mais pérenne entre le délire pré-rationnel de haine de l'autre et la fonctionnalisation instrumentale la plus aveugle de la science ont pu produire tant Hiroshima qu'Auschwitz, il convient à la fois de ne pas accéder à la prétention de la théorie à se prendre trop au sérieux, tout en étant conscient que c'est pourtant aussi avec elle qu'on peut sortir de la barbarie. La voie ainsi dessinée, sur un fil, impose à la Raison d'intégrer dialectiquement, dans le travail intellectuel de production de la théorie, tout ce à quoi elle s'oppose dans le mouvement par lequel elle se constitue : les qualités, le subjectif, l'imagination, tout ce qui ne s'identifie pas par avance à aucune structure d'ensemble. Parmi les théoriciens qu'Adorno respectait, Siegfried Kracauer, son ami d'enfance, est selon lui un exemple de ceux qui ont réussi à porter cette exigence jusqu'au plus fort du travail de théorisation : 
« Dans tous ses travaux, Kracauer rappelle que la pensée, en regardant derrière soi, ne doit pas oublier ce dont elle s'est nécessairement débarrassée pour devenir une pensée. Ce moment est matérialiste; il amena Kracauer, presque malgré lui, à la critique de la société, dont l'esprit s'emploie avec zèle à un tel oubli. En même temps, le refus de la pensée absolument abstraite vient faire obstacle à la logique matérialiste. ${ }^{56}$

Ensuite, il convient de rendre compte de ces tensions qui traversent le social dans une forme d'écriture qui puisse y donner accès le plus possible. Il s'agit de ne pas voiler ou lisser les contradictions derrière le vernis des normes et attendus scientifiques tacites, qui sont souvent une limitation de toute intention critique dans la rédaction des articles scientifiques. Pour Adorno, c'est l'essai, comme forme de la production théorique - forme que ses amis Kracauer et surtout Walter Benjamin ont particulièrement éprouvée -, qui matérialise le mieux cette exigence, tant dans la production théorique esthétique que philosophique ou sociologique :

"L'essai a été presque le seul à réaliser dans la démarche même de la pensée la mise en doute de son droit absolu. Sans même l'exprimer, il tient compte de la nonidentité de la conscience ; il est radical dans son non radicalisme, dans sa manière de s'abstenir de toute réduction à un principe, de mettre l'accent sur le partiel face à la totalité dans son caractère fragmentaire. ${ }^{57}$

C'est bien l'essai, dans son rapport particulier à l'expérience qu'il cherche à décrire, et à propos de laquelle il s'autorise l'interprétation, forcément médiate, subjective et parcellaire, qui est susceptible de porter l'engagement d'un travailleur intellectuel qui cherche à maintenir le travail de la théorie à la hauteur de son propre concept :

« Il prend la logique hégélienne au mot: il ne faut ni brandir la vérité de la totalité contre les jugements particuliers, ni limiter la vérité au jugement particulier, mais prendre à la lettre la prétention de la singularité à être vraie, jusqu'à ce que sa nonvérité devienne une évidence. Tout ce qu'il y a de risqué, de prématuré, de pas tout à fait garanti dans chacun des détails de l'essai entraîne d'autres détails qui en sont la négation ; la non-vérité dans laquelle l'essai s'enfonce en connaissance de cause est l'élément de sa vérité. $\|^{58}$

L'essai est ainsi le lieu de la saisie du particulier, hors de toute limite ou formatage dus à la segmentation disciplinaire. Les récentes publications des dialogues entre Adorno et Horkheimer, tant en vue de l'écriture de leur ouvrage à quatre mains ${ }^{59}$, que d'un «nouveau manifeste communiste » (qui n'a finalement jamais été achevé) ${ }^{60}$ mettent en évidence leur souci commun - quelquefois aliéné par des contraintes éditoriales - de toujours laisser au maximum transparaître dans leur écriture ce caractère contradictoire et non-réconcilié des réalités à décrire. Plusieurs passages montrent ainsi combien le travail de et sur l'écriture, typique de cet « essayisme » qui caractérise la forme interdisciplinaire des travaux de l'École de Francfort à partir de la Dialectique de la raison ${ }^{61}$, est d'une importance primordiale, irréductible aux contraintes et normes scientifiques institutionnalisées par les habitudes disciplinaires. Il s'agit d'approcher au plus près et de la manière la plus explicite qui soit, dans la manière de rendre compte d'une analyse, les contradictions tant internes à cette dernière que révélées par elle.

Voilà donc comment la possibilité d'un engagement du scientifique comme de l'artiste, peut apparaître aux théoriciens critiques comme tâchant de se hisser à la hauteur de la catégorie de travail intellectuel. Le travail intellectuel, qui porte en lui le souvenir d'une histoire de la domination qui contient aussi celui d'une époque pré-capitaliste où la société industrielle n'avait pas soumis toute activité de l'esprit à la loi de la marchandise, doit être conservé à tout prix comme la contradiction qu'il représente et 
qui rappelle qu'il pourrait en être autrement. Tout individu qui s'adonne à un travail intellectuel, même dans une situation enserrée dans les rapports de production industriels peut retrouver, au moins par interstices, cette attitude de méditation qui a pu caractériser le travail intellectuel lors de sa séparation d'avec le travail manuel. À partir de ce sentiment, éprouvé en filigrane, la pratique fondamentale du travailleur intellectuel digne de ce nom doit être une pratique permanente de lutte contre l'industrie culturelle qui enserre et vise à réduire à néant la force intimement critique de sa production intellectuelle, critique parce que justement non soumise a priori. Cette tâche, exigeante, est toujours susceptible de s'abîmer en son contraire (le divertissement, la pensée positive, etc.) mais doit toujours, contre les forces qui concourent à sa fonctionnalisation maintenir ses espaces de négation de ce-qui-est au sein de ce-qui-est.

\section{Ouverture}

Ayant en tête les mouvements actuels et le bouillonnement théorique qu'ils ont fait naître (et auquel cette livraison de la revue est consacrée), il apparaît toutefois que le présent article ne peut en rester là. Car en admettant que «la culture [activité théorique comme artistique] est le fruit de la séparation radicale entre travail manuel et travail intellectuel et tire ses forces de cette séparation qui est en quelque sorte son péché originel », et en acceptant également que "[l]orsque la culture se contente de renier cette séparation en donnant l'illusion d'une solidarité directe, elle reste en dessous de son propre concept ${ }^{62}$, il n'en demeure pas moins que les théoriciens ne peuvent se satisfaire de cette démonstration (voire la maintenir comme excuse de leur propre désengagement ${ }^{63}$ éventuel de la pratique politique) qu'au risque de voir leur production retomber dans une glose de classe à tout moment susceptible d'être rattrapée par la logique de l'industrie culturelle. On aurait tort, cependant, d'interpréter alors - comme c'est souvent le cas - la position d'Adorno notamment, comme un simple renoncement, doublé d'un repli dans l'esthétique. Il s'agit plutôt, dans le cas du philosophe francfortois, de la manifestation d'une volonté à laquelle il a consacré son existence, de tenir à tout prix la possibilité d'une expérience de travail intellectuel, dans un monde post-Seconde Guerre mondiale où l'emprise de l'industrie culturelle et le pouvoir des faits, ainsi que les totalitarismes à l'Est rivalisant alors d'imagination avec le nazisme pour décimer les intellectuels, se sont unis dans une entreprise de destruction de la pensée. Et si, pour rester sur Adorno, on peut remarquer plusieurs contradictions entre certaines de ses positions tant théoriques que pratiques - aboutissant au célèbre chahut dont il a fait l'objet en 1968 de la part de ses étudiant(e)s exhortant la Théorie critique dont il était représentant à s'engager dans la pratique politique immédiate et lors duquel il a fait expulser ceux(celles)-ci par les forces de l'ordre -, il ne nous semble pas pertinent ni de mettre ces contradictions en avant comme limite absolue et impuissance finale de la théorie adornienne, ni de chercher à les dissimuler ou à les réduire. En effet, la première proposition révélerait une logique qui tend à identifier de manière bien peu dialectique contradiction et erreur, à la manière des théories positivistes que la Théorie critique combat; la seconde reviendrait à un lissage neutralisant le caractère le plus fondamentalement critique d'Adorno - et Alexander Neumann rappelle combien cette position tend à être celle de ses est celle de ses "héritiers légaux et symboliques", en particulier de Jürgen Habermas ${ }^{64}$. Nous considérons qu'il est bien plus stimulant de considérer ces 
contradictions comme l'expérience faite par Adorno de l'impossibilité de la réconciliation ${ }^{65}$. Cette expérience, vivante et qui n'est pas sans rappeler le thème de la Dialectique négative, devrait ainsi être au point de départ d'une réflexion sur le travail intellectuel à partir de la Théorie critique aujourd'hui. Ce n'est pas avec une version muséifiée - et ainsi rendue dans le même temps mutique - de cet ancrage théorique que nous voudrions inviter à travailler mais plutôt avec une Théorie critique active et exigeante, telle qu'elle s'est constituée notamment dans les travaux d'Adorno, aux prises avec les catastrophes et constats d'échec des deuxième et troisième quarts du $\mathrm{XX}^{\mathrm{e}}$ siècle qu'elle a traversés. S'il s'agissait donc, pour terminer cette excursion théorique, d'ouvrir et proposer à débat quelques réflexions, en discutant également certaines propositions actuelles quant à la catégorie du travail intellectuel, en rapport tant avec la marche de la logique du capitalisme et de l'industrie culturelle que des mouvements de lutte, c'est bien à partir de cette contradiction dont Adorno et certains autres théoriciens critiques ont fait l'expérience vivante, témoignant d'un éloignement de la possibilité de la réconciliation entre l'exigence toujours maintenue du travail intellectuel avec les forces sociales, que nous voudrions avancer. Il nous semble alors absolument nécessaire de garder à l'esprit l'opposition que forme la catégorie de travail intellectuel avec le concept d'industrie culturelle, qui cherche toujours à le réintégrer, le rendre inoffensif. Et il nous semble que plusieurs propositions actuelles qui se donnent pour objet de penser les mouvements contemporains et éventuellement le rôle des intellectuels en leur sein (théoriciens, mais également artistes) tendent à oublier ce rapport.

31 Par exemple, dans son très récent et stimulant essai Pour un suicide des intellectuels, le politiste Manuel Cervera-Marzal propose une dissolution de la catégorie et du statut d'intellectuel. Il ne s'agit bien évidemment pas d'un appel à une liquidation barbare des personnes engagées dans le travail intellectuel, mais plutôt d'une réflexion posant « la perspective d'une disparition des intellectuels en tant que catégorie sociale distincte du reste de la population ${ }^{66}$. Rappelant que l'intellectuel - i.e. tout individu dont la profession ressortit de la catégorie du travail intellectuel - « doit [...] admettre que sa position est un privilège enviable dont il jouit rarement en vertu de ses propres mérites " ${ }^{67}$ l'auteur invite ce dernier à militer pour "poursuivre la démocratisation de la fonction d'intellectuel tout en redistribuant simultanément les tâches manuelles " ${ }^{68}$. Alors, poursuit Cervera-Marzal, "[c]e bouleversement économique s'accompagnerait d'une égalisation des salaires et mettrait un terme à la supériorité du travail intellectuel sur le travail manuel. Au fond, dans une telle société, la distinction entre les travailleurs manuels et intellectuels aurait disparu ${ }^{69}$. Cet appel nous semble important, qui rappelle combien la possibilité de s'engager dans les activités intellectuelles - comprenant les activités artistiques - ne devrait en aucun cas demeurer un privilège mais une possibilité offerte à tout individu, à la faveur d'une redistribution du travail qui, réduisant drastiquement le temps du travail accompli pour la subsistance, permettrait à chacun le développement de ses capacités cognitives et artistiques. Mais cet essai, qui entend renouer avec une proposition marxienne assez classique, ne nous paraît justement pas prendre assez la mesure de l'industrie culturelle dont nous avons rappelé les développements quant à la catégorie du travail intellectuel.

Ainsi, quand l'auteur rappelle le problème d'une institution universitaire qui " génère un morcellement de notre compréhension du monde puisqu'elle isole les disciplines les 
unes des autres $»^{70}$ il ne semble pas lier cette tendance à la logique générale de la division du travail qui, ne se satisfaisant pas de la simple séparation du travail manuel et intellectuel a, dans les mutations contemporaines du capitalisme, fait de la division un principe, tant pour les tâches manuelles de la production que pour celles de la pensée ou de la production artistique, culturelle et informationnelle. Il nous paraît au contraire nécessaire d'interpréter ce mouvement d'hyperspécialisation et de cloisonnement disciplinaire comme l'un des éléments de liquidation de la pensée par sa réduction à des procédures spécialisées dans l'industrie culturelle, au même titre (et dans le même temps) que la mise en concurrence de plus en plus acharnée des chercheurs quant à l'obtention des financements pour la recherche, dans une logique de projet toujours plus teintée de nécessité bureaucratique ${ }^{71}$ que nous avons discutée supra. Et le simple partage du travail intellectuel auquel appelle l'auteur n'est en rien un gage de fonctionnement plus ouvert des lois de toute institution de production médiatique ou théorique acquise à l'industrie culturelle. On peut même imaginer ici au contraire que ce partage pourrait être un point de justification extrême de l'hyperspécialisation car l'idée que "[1]e travail en équipe favorise l'accumulation de connaissance et l'élargissement du regard $\aleph^{72}$ est aussi l'argument avancé pour légitimer qu'on ne s'attaque pas au morcellement des savoirs : chacun au sein d'une équipe vient apporter son savoir issu de son parcours intellectuel propre; un intellectuel plus en vue que les autres sera invité à produire la synthèse issu des discussions fructueuses de l'équipe et pourra justifier de présenter un autre $\operatorname{projet}^{73}$. Il nous semble ainsi qu'en rappelant la nécessité du partage du travail comme de la critique de la division du travail (avec lesquelles nous sommes entièrement d'accord) l'auteur ne pose pas assez la question de ce que serait le travail intellectuel à partager dans un monde actuellement baigné d'industrie culturelle: tout semble se passer comme si ce partage était déjà effectif, ne fussent les intellectuels occupés à se maintenir l'exclusivité du travail de la pensée. Si nous agréons totalement avec Cervera-Marzal que « l'excellence de la pensée ne s'oppose pas à sa démocratisation " $^{74}$, il nous apparaît nécessaire de rappeler que, démocratisation ou non, l'industrie culturelle actuelle, et ses manifestations tant dans la culture que l'éducation et la production théorique, s'oppose, elle, à cette excellence de la pensée. Il ne faudrait donc pas que la quête d'un partage plus équitable et rationnel des tâches et notamment des tâches intellectuelles fasse oublier la lutte nécessaire contre les mouvements de l'industrie culturelle - sans quoi ces tâches intellectuelles risquent de s'abîmer à leur tour en pures redites de l'appareil de production.

Il est bien certain que, si elle est un but de tout projet communiste, la réunion du travail manuel et du travail intellectuel ne se décrète pas. Il en est de même pour la réconciliation de la théorie universitaire et de la pratique militante qui en est un premier moment important, tant chez Cervera-Marzal que chez un certain nombre d'autres chercheurs ou militants ${ }^{75}$. Il y a là la continuité d'une certaine considération des rapports entre théorie sociale et pratique politique comme ayant nécessairement cette dernière pour but et arbitre de la vérité de la théorie, qui voit dans la séparation des théoriciens critiques des cœurs de la stratégie politique un péché originel du marxisme " occidental $»^{76}$ dont les premières figures paradigmatiques sont, d'après Perry Anderson, les théoriciens de l'École de Francfort. Mais il ne suffit pas de décider que cette séparation de la théorie et de la pratique, fruit du mouvement de la division du travail, est simplement désormais nulle et non avenue - ce qui n'empêche pas qu'il faille encourager les expériences qui tendent à la dépasser, comme autant de brèches. 
34 À ce propos, d'autres appels, pourtant plus proches du cadre théorique de la Théorie critique, et tout en s'éloignant d'un marxisme identifiant trop rigidement théorie et stratégie, ne paraissent pas non plus accorder assez d'importance à la problématique de l'industrie culturelle, ni poser la question du penser ou de la production artistique comme « travail intellectuel ». C'est le cas, par exemple, des vivifiantes 33 thèses contre le capital de John Holloway, traduites en français en $2012^{77}$. À partir des expériences quotidiennes, ordinaires et plus ou moins structurées et concertées de refus et de rejet de la vie imposée par le capitalisme (de celle de l'employée de bureau qui déserte un matin son poste pour aller s'asseoir dans un parc à celle du groupe d'ouvriers industriels qui occupent et font tourner leur usine en autogestion, en passant par un groupe de sans-abri refusant de payer le loyer d'une maison qu'ils occupent, etc.), l'auteur invite à reconsidérer la révolution comme un "processus interstitiel »: « [1]a seule façon de penser le changement radical du monde est de le concevoir comme la multiplicité de mouvements interstitiels découlant du particulier $»^{78}$. Pour Holloway, il s'agit de considérer le caractère double de l'activité humaine - le " faire » - depuis son versant irréductible à la loi de la valeur et de sa transformation en travail abstrait, et de se concentrer sur un ensemble de manifestations particulières de cette irréductibilité dans le monde contemporain en crise. Ces manifestations, que l'auteur analyse comme autant de «brèches» fragiles dans le mur du capitalisme, par l'expression «ici et maintenant » d'aspirations à une vie au-delà des limitations imposées par la loi de la valeur, doivent être élargies et s'étendre en cascade, au-delà des visions traditionnelles des luttes qui limitent leur portée aux engagements objectifs et raisonnés de groupes d'activistes au sein de catégories de populations identifiées comme sujets porteurs de changement. «Le changement social est plutôt le résultat de la transformation à peine visible de l'activité quotidienne de millions de personnes. Nous devons regarder au-delà de l'activisme et donc vers les millions et millions de refus et d'autres-faire, les millions et millions de fissures qui constituent le matériel de base d'un possible changement radical. $\aleph^{79}$ Chaque brèche, par laquelle le faire des individus s'oppose au travail abstrait, au temps de travail socialement nécessaire selon les règles qu'impose le capitalisme, vise ainsi l'établissement de nouveaux rapports interindividuels s'opposant aux rapports marchands réclamant en permanence également de nouvelles formes d'organisation selon «un processus incessant d'expérimentation de formes démocratiques, de façon de surmonter les inhibitions des gens, de façons de contrôler leur agressivité ou leurs visées sexistes ou racistes $»^{80}$. Ces expériences sont à étendre, non parce qu'elles seraient des moments d'une marche vers l'avènement d'un monde autre, mais parce qu'elles sont déjà, "ici et maintenant", des bribes de cet autre monde : «En fait, chaque grève crée de nouveaux rapports d'amitié et de solidarité, et donne aux grévistes l'expérience pratique d'un monde sans patrons : la création d'un monde avec des rapports sociaux différents va au-delà de ce qui était prévu lors du déclenchement du conflit. $~^{81}$

Si une catégorie comme celle du faire a comme mérite fondamental de rappeler que c'est par l'activité des individus, qui excède largement les limites des activités entendues dans le seul cadre du travail dans le capitalisme, que le changement se produit - d'où l'importance de la prise de conscience subjective de la puissance d'agir individuelle de chacun à laquelle l'ouvrage exhorte -, et si, chez Holloway, elle rend particulièrement fluides et saturés d'expérience des concepts ailleurs très abstraits, elle tend aussi à simplifier la problématique de la division du travail en se contentant de la nier. Le faire contient à la fois le savoir et les activités intellectuelles qui semblent avoir 
déjà mis leur ouverture d'esprit et leur créativité du côté de la lutte, même s'il ne peuvent qu'avouer leur relative impuissance quant à la proposition de "recettes à appliquer " qui, dialectiquement, ne feraient de toute façon que produire "une structure organisationnelle différente, une structure de monologue qui instaure des dirigeants et des institutions pour les mettre en place ${ }^{82}$. Ainsi, le travail intellectuel n'est posé que comme cas particulier du travail, dans son caractère double : une partie du travail intellectuel n'existe que limitée dans le cadre de la création de la valeur, mais en tant que faire individuel ou partagé, il se déploie au-delà de ces limites. Derrière une telle catégorie, c'est bien, à nouveau, la problématique de l'industrie culturelle qui tend à être oubliée : cette entreprise de limitation de ce qui historiquement s'est autorisé à dépasser les limites du toujours identique par sa séparation des tâches routinières et de dur labeur. C'est aussi un oubli de l'industrie culturelle comme travail intellectuel employé à (re)produire de l'identique et de l'adéquat à partir des sentiments d'inadéquation et des consciences d'inadaptations individuelles qui sont justement, pour Holloway, à l'origine des brèches. Il ne faudrait pas oublier, en effet, combien à l'heure actuell, ces aspirations à « l'émergence [...] d'un autre monde, d'un autre faire et d'autres relations $»^{83}$ sont réintégrées - voire servent carrément à régénérer et réactualiser les imaginaires contemporains de la forme-marchandise. Ainsi, lorsque Holloway rappelle qu' « il y a de réelles pressions (la répression, la famine) qui nous poussent à reproduire le capitalisme chaque jour ${ }^{84}$, il nous semble qu'il devrait $\mathrm{y}$ ajouter un certain nombre de pratiques culturelles ou médiatiques dont il a par ailleurs, pour sa part, choisi d'interpréter certaines comme des «brèches » qui visent justement à cesser de fabriquer le capitalisme. Et lorsqu'il incite à briser « les murs autour de notre esprit qui rigidifient notre pensée, un processus qui résulte du travail abstrait et qui est renforcé dans les écoles et dans les universités » ou " les murs par lesquels la dynamique puissante des verbes est enfermée par les noms " ${ }^{85}$ c'est bien à une tâche de résistance $\mathrm{du}$ travail intellectuel à l'industrie culturelle, et son mouvement d'intégration constant de tout embryon de pensée contradictoire, qu'il appelle. En plus d'appeler à une convergence de ces nouvelles pratiques, de ces brèches, Holloway devrait alors aussi appeler à la constitution d'une pensée spécifique et commune des brèches, qui ne se fondrait jamais complètement dans le faire qu'à la condition d'être rattrapée par l'industrie culturelle et l'illusion de la réconciliation par pure identification avec ce qui est.

Si le travail intellectuel s'oppose, dans sa forme, au travail de la praxis (des mobilisations sociales par exemple, ou des nouvelles activités ouvertes par de nouvelles pratiques), il n'est en aucun cas une opposition des personnes susceptibles de s'engager dans l'un comme dans l'autre. Ces dernières peuvent ainsi, bien évidemment, être les mêmes, à l'heure où la possibilité d'une réconciliation ne semble pour le moment pas à l'ordre du jour - ce qui n'enlève rien à la nécessité de conserver à l'esprit tous les termes de ce qui ne se réconcilie pas. Il est ainsi à notre avis important de garder cet appel au partage du travail intellectuel, comme des autres tâches, des autres pratiques qui peuvent, à des degrés divers, représenter des «embryons » de libération. L'hypothèse que nous aimerions alors livrer à discussion pour finir est que ce n'est peut-être qu'à la faveur d'une lutte partagée, entre des aspirations à faire naître d'autres expériences par la transformation des pratiques sociales, par exemple dans la mobilisation ou dans 
la production d'autres faires, d'un côté, et des aspirations à maintenir les conditions exigeantes d'une activité théorique et artistique, de l'autre, sans nier l'opposition fondamentale des deux, mais en prenant conscience, au contraire, que cette opposition est la matérialisation de ce que la société actuelle et telle qu'elle nous est donnée est cet ensemble irréconcilié que, d'une manière assez paradoxale et en négatif, une réconciliation peut se dessiner. Le lieu de cette réconciliation ne serait alors ni la pratique sociale et de mobilisation, ni le travail intellectuel de production de la théorie mais le sujet, susceptible de s'engager dans l'une et dans l'autre. Ce n'est toutefois qu'une réconciliation décevante et en forme d'opposition continuelle: il s'agit en réalité plutôt d'une intégration forcée des tendances oppositionnelles contradictoires par le sujet. Engagé tant dans une pratique de mobilisation sociale ou de pratique alternative que dans un travail théorique qui maintient son exigence, l'individu ne peut qu'espérer faire au niveau le plus intime de sa subjectivité l'expérience fondamentale de la société avancée : celle de la contradiction entre ces deux pôles qui ne se réunissent $a$ priori ni directement dans le faire, ni dans une hiérarchie à inverser entre la pratique sociale et la production théorique.

Il convient alors d'exacerber les mouvements et mobilisations de la critique sociale et d'encourager la prise de conscience qu'une autre société doit être atteinte par la pratique, en "refusant de continuer à fabriquer le capitalisme" comme y invite Holloway. Il s'agit d'exalter l'intuition que font de plus en plus d'individus, dans les secteurs les plus divers de la production sociale, que l'expérience quotidienne qu'ils peuvent faire de la souffrance au travail, du mépris, etc. est due à une logique capitaliste barbare qui ne se dissimule même plus et qui voit tous les jours se réduire le nombre de ses bénéficiaires (mais augmenter leurs parts de bénéfices). Cette pratique de la lutte sociale ne doit pas pour autant voir dans les productions théoriques un idéal de réconciliation qui devrait diriger la pratique dans l'immédiat. Mais, alors que le travail théorique ne peut plus viser sa réconciliation immédiate avec une pratique, il reste le lieu fondamental d'une activité oppositionnelle dans l'expérience subjective même des individus. Il convient donc aussi de s'engager pour limiter la fonctionnalisation toujours plus prégnante des lieux de production du travail théorique, critiquer la casse de l'université, et toutes les logiques qui concourent à maintenir le travail de la théorie en-deçà de son propre concept de théorie - et qui sont les mêmes que celles qui, ailleurs, exproprient toujours plus le travail «nonintellectuel ». Il faut également encourager le plus d'individus possible à y prendre part, à la faveur notamment de luttes pour une réduction drastique du temps de travail, comme y invite Cervera-Marzal, ainsi que pour une gratuité effective de l'accès à l'enseignement supérieur et à l'enseignement artistique. Ce travail théorique ou artistique, toujours partageable, et qu'il convient de partager davantage, libérant ainsi le «flux dans lequel le savoir d'une personne se mêle et se mélange de façon indéfinissable au savoir des autres ${ }^{86}$, révèle aussi au sujet qui le produit, le partage ou le reçoit, à quel point la pratique dans laquelle il est engagé par ailleurs dans ses autres activités est à son tour en-deçà de ce qu'elle devrait être eu égard aux potentialités ouvertes par la technique et le niveau scientifique et philosophique que l'époque a atteint - soit, en termes adorniens que « tout est faux aussi longtemps que le monde est comme il est $\aleph^{87}$. Tout le monde doit pouvoir, à son niveau, faire cette expérience, qui est fondamentalement celle que l'industrie culturelle vise au contraire à empêcher. De là, il n'est pas sûr que le salut soit absolument dans un dépassement par la pratique pure ni par la théorie pure, mais probablement dans une lutte menée à des niveaux 
différents. Pour le moment, en revanche, il nous apparait certain que ce n'est pas en niant purement et simplement la séparation des deux que la critique avance : cette simple négation "théorique " de la division du travail ne fait pour autant pas cesser d'exister les réalités que cette division a fait naitre historiquement. L'industrie culturelle, fruit de cette division, s'emploie encore et toujours à se présenter au contraire comme réconciliation. Ne pas faire son jeu, c'est aussi reconnaître la particularité du travail intellectuel, le mieux placé dans sa pratique de travail pour la combattre. Cela qui ne signifie pas qu'il faille pour autant réserver ce travail à une catégorie réduite d'individus, mais inversement, l'ouverture de ce travail ne peut signifier qu'il cesse d'exister comme catégorie spécifique - sauf à capituler par avance.

\section{BIBLIOGRAPHIE}

Adorno, Theodor W. « Arnold Schönberg ». In Prismes : critique de la culture et société, 151-79. Paris: Payot, 1986.

Adorno, Theodor W. « Capitalisme tardif ou société industrielle?» In Société: IntégrationDésintégration. Écrits sociologiques, 85-107. Paris: Payot, 2011.

Adorno, Theodor W. Current of music. Éléments pour une théorie de la radio. Paris: Maison des sciences de l'homme, 2010.

Adorno, Theodor W. « Engagement ». In Notes sur la littérature, 285-306. Paris: Flammarion, 1984.

Adorno, Theodor W. Le caractère fétiche dans la musique et la régression de l'écoute. Paris : Allia, 2001.

Adorno, Theodor W. « Le teamwork en recherche sociale ». In Le conflit des sociologies. Théorie critique et sciences sociales, 305-11. Paris: Payot, 2016.

Adorno, Theodor W. «L'essai comme forme ». In Notes sur la littérature, 5-29. Paris: Flammarion, 1984.

Adorno, Theodor W. « Mode intemporelle ». In Prismes : critique de la culture et société. Paris : Payot, 1986.

Adorno, Theodor W. Théorie esthétique. Paris : Klincksieck, 1995.

Adorno, Theodor W. Trois études sur Hegel. Paris: Payot, 1979.

Adorno, Theodor W. «Un étrange réaliste : Siegfried Kracauer ». In Notes sur la littérature, 263-83.

Paris: Flammarion, 1984.

Adorno, Theodor W., et Hanns Eisler. Musique de cinéma. Paris : l'Arche, 1972.

Adorno, Theodor W., et Max Horkheimer. Towards a new manifesto. London: Verso, 2011.

Adorno, Theodor W., et Karl Popper, éd. De Vienne à Francfort. La querelle allemande des sciences sociales. Bruxelles: Éditions Complexe, 1979.

Anderson, Perry. Sur le marxisme occidental. Paris: Maspéro, 1977. 
Bensaïd, Daniel, et Philippe Corcuff. « Le travail intellectuel au risque de l'engagement ». Revue Agone, $\mathrm{n}^{\circ}$ 18-19 (1998). http://danielbensaid.org/Le-travail-intellectuel-au-risque\#nh1.

Bonefeld, Werner. Critical Theory and the Critique of Political Economy. New-York, NY: Bloomsbury, 2014.

Cervera-Marzal, Manuel. Pour un suicide des intellectuels. Paris: Textuel, 2016.

Ernaux, Annie. L'écriture comme un couteau. Entretien avec Frédéric-Yves Jeannet. Paris: Stock, 2003. Granger, Christophe. La destruction de l'université française. Paris: La Fabrique, 2015.

Holloway, John. Crack capitalism. 33 thèses contre le capital. Paris: Libertalia, 2012.

Horkheimer, Max. Éclipse de la raison. Paris: Payot, 1974.

Horkheimer, Max. Théorie critique. Paris : Payot, 1978.

Horkheimer, Max. « Théorie traditionnelle et théorie critique ». In Théorie traditionnelle et théorie critique, 15-81. Paris : Gallimard, 1974.

Horkheimer, Max, et Theodor W. Adorno. La dialectique de la raison. Paris: Gallimard, 1974.

Horkheimer, Max. Le laboratoire de la Dialectique de la raison. Discussions, notes et fragments inédits. Paris: Maison des sciences de l'homme, 2013.

Krahl, Hans-Jürgen. Konstitution und Klassenkampf. Zur historischen Dialektik. Von bürgerlicher Emanzipation und proletarischer Revolution. Frankfurt am Main: Verlag Neue Kritik, 1971.

Laudani, Raffaele. « Pour une théorie critique de l'indignation. Notes sur le rapport actuel entre les mouvements sociaux et le pouvoir destituant ». Illusio, nº 10-11 (2013), 97-105.

Magis, Christophe. «Art, culture et critique en communication : propositions pour une théorie des industries culturelles ». In Critique, sciences sociales et communication, édité par Éric George et Fabien Granjon, 137-64. Paris: Mare \& Martin, 2014.

Marcuse, Herbert. La dimension esthétique. Paris: Seuil, 1979.

Marcuse, Herbert. L'homme unidimensionnel. Paris : Minuit, 1968.

Marcuse, Herbert. « Note sur la dialectique ». In Raison et révolution. Hegel et la naissance de la théorie sociale. Paris: Minuit, 1968.

Marx, Karl, et Friedrich Engels. L'idéologie allemande. Paris: Editions Sociales, 1976.

Matonti, Frédérique, et Gisèle Sapiro. « L'engagement des intellectuels : nouvelles perspectives ». Actes de la recherche en sciences sociales $\mathrm{n}^{\circ} 176-177, \mathrm{n}^{\circ} 1$ (2009), 4-7.

Neumann, Alexander. « Le courant chaud de l'École de Francfort ». Variations. Revue internationale de théorie critique, $\mathrm{n}^{\circ} 12$ (2008).

Neumann, Alexander. « Pour une écoute sociologique. Répression, inhibition, prise de parole ». Variations. Revue internationale de théorie critique, $\mathrm{n}^{\circ} 8$ (2006).

Noiriel, Gérard. Dire la vérité au pouvoir. Les intellectuels en question. Marseille: Agone, 2010.

Piponnier, Anne. « Le projet dans les pratiques de recherche. Pour un retour réflexif et critique sur nos engagements ». Sciences de la société, nº 93 (2015), 110-23.

Postone, Moishe. Temps, travail et domination sociale. Paris: Fayard, 2009.

Raulet, Gérard. Herbert Marcuse. Philosophie de l'émancipation. Paris: PUF, 1992. 
Raulet, Gérard. « Interdisciplinarité ou essayisme ? La "philosophie sociale” de la Dialektik der Aufklärung ». In Jenseits instrumenteller Vernunft. Kritische Studien zur "Dialektik der Aufklärung », édité par Manfred Gangl et Gérard Raulet. Berlin: Peter Lang, 1998.

Sapiro, Gisèle. La responsabilité de l'écrivain. Littérature, droit et morale en France (XIXe-XXIe siècle). Paris: Seuil, 2011.

Sartre, Jean-Paul. Qu'est-ce que la littérature ? Paris: Gallimard, 1948.

Sohn-Rethel, Alfred. La pensée-marchandise. Bellecombe-en-Bauge: Éditions du croquant, 2010.

Voirol, Olivier. « Retour sur l'industrie culturelle ». Réseaux 29, nº 166 (2011), 25-57.

\section{NOTES}

1. Cf. par ex. Gérard Noiriel, Dire la vérité au pouvoir. Les intellectuels en question (Marseille: Agone, 2010); Frédérique Matonti et Gisèle Sapiro, «L'engagement des intellectuels: nouvelles perspectives ", Actes de la recherche en sciences sociales $n^{\circ} 176-177,{ }^{n} 01$ (2009) ; Daniel Bensaïd et Philippe Corcuff, "Le travail intellectuel au risque de l'engagement», Revue Agone, no 18-19 (1998), http://danielbensaid.org/Le-travail-intellectuel-au-risque\#nh1. Nous reviendrons notamment, dans la dernière partie de ce texte, sur l'ouvrage de Manuel Cervera-Marzal, Pour un suicide des intellectuels (Paris: Textuel, 2016).

2. Voir l'article de Fabien Granjon dans ce numéro.

3. Cf. ci-après, quatrième section de l'article.

4. Cf. Dietrich Hoss, «La misère de la Théorie critique... est le manque de la question de l'organisation", Illusio, ${ }^{\mathrm{n}} \mathrm{O}$ 10-11 (2013), 76-95. On pourra également consulter le principal ouvrage de Hans-Jürgen Krahl, Konstitution und Klassenkampf. Zur historischen Dialektik. Von bürgerlicher Emanzipation und proletarischer Revolution (Frankfurt am Main: Verlag Neue Kritik, 1971).

5. Quand ses collègues Adorno et Horkheimer sont revenus en Allemagne dans les années 1950.

6. Laudani, «Pour une théorie critique de l'indignation. Notes sur le rapport actuel entre les mouvements sociaux et le pouvoir destituant $", 83$.

7. Cf. Gérard Raulet, Herbert Marcuse. Philosophie de l'émancipation (Paris: PUF, 1992).

8. Laudani, «Pour une théorie critique de l'indignation. Notes sur le rapport actuel entre les mouvements sociaux et le pouvoir destituant».

9. Cf Werner Bonefeld, Critical Theory and the Critique of Political Economy (New-York, NY : Bloomsbury, 2014) ; Moishe Postone, Temps, travail et domination sociale (Paris: Fayard, 2009) ou le numéro 10/11 de la revue Illusio, ayant pour thème "Théorie critique de la crise: École de Francfort, controverses et interprétations » (2013).

10. On consultera pour s'en convaincre le texte fondateur de Max Horkheimer, "Théorie traditionnelle et théorie critique ", in Théorie traditionnelle et théorie critique (Paris: Gallimard, 1974), 15-81, où les références à la division du travail, et notamment du travail scientifique, sont très nombreuses.

11. Cf. Alfred Sohn-Rethel, La pensée-marchandise (Bellecombe-en-Bauge : Éditions du croquant, 2010).

12. Max Horkheimer et Theodor W. Adorno, La dialectique de la raison (Paris : Gallimard, 1974), 31.

13. Ibid.

14. Max Horkheimer, Éclipse de la raison (Paris : Payot, 1974), 62-63.

15. Horkheimer et Adorno, La dialectique de la raison, 50.

16. Theodor W. Adorno, Trois études sur Hegel (Paris : Payot, 1979), 32. 
17. Les Minima Moralia d'Adorno comme les Notes critiques de Horkheimer sont à cet égard assez instructifs.

18. Max Horkheimer, Théorie critique (Paris : Payot, 1978), 327.

19. Karl Marx et Friedrich Engels, L’idéologie allemande (Paris : Editions Sociales, 1976), 30.

20. Cf. Adorno, Trois études sur Hegel, 30. sq.

21. Theodor W. Adorno et Max Horkheimer, Towards a new manifesto (London : Verso, 2011), 95.

22. Cf. à ce sujet Albrecht Wellmer, « Autonomie et négativité de l'art. L'actualité de l'esthétique d'Adorno et les points aveugles de sa philosophie de la musique ", Réseaux, vol. 29, nº 166 (2011), 31-70.

23. Cf. par ex. Herbert Marcuse, "Note sur la dialectique", in Raison et révolution. Hegel et la naissance de la théorie sociale (Paris : Minuit, 1968), 45.

24. Cf. par exemple Christophe Magis, Nelly Quemener et Florian Vörös (dir.), "Exploitation 2.0", Poli-Politique de l'image, $\mathrm{n}^{\circ} 13$.

25. Horkheimer et Adorno, La dialectique de la raison, 22.

26. Et bien que cette analyse et les questions de planification industrielle, de standardisation, de contrôle et d'accumulation capitalistique appliqués à la production culturelle y soient absolument centrales, cf. Christophe Magis, «Art, culture et critique en communication: propositions pour une théorie des industries culturelles", in Critique, sciences sociales et communication, éd. par Éric George et Fabien Granjon (Paris : Mare \& Martin, 2014), 137-64. ; voir aussi Olivier Voirol, « Retour sur l'industrie culturelle », Réseaux, ${ }^{\mathrm{n}} \mathrm{O} 166$ (2011), 125-57.

27. Theodor W. Adorno, Current of music. Éléments pour une théorie de la radio (Paris : Maison des sciences de l'homme, 2010).

28. Theodor W. Adorno, "Mode intemporelle», in Prismes : critique de la culture et société (Paris : Payot, 1986).

29. Theodor W. Adorno et Hanns Eisler, Musique de cinéma (Paris : l'Arche, 1972).

30. Theodor W. Adorno, Le caractère fétiche dans la musique et la régression de l'écoute (Paris : Allia, 2001), 16. Si cet essai est antérieur à la rédaction de la dialectique de la Raison et donc à la formulation du concept d'industrie culturelle, il n'en est pas moins l'une des premières tentatives de cette formulation, à partir d'une référence à la théorie du fétichisme de la marchandise de Marx.

31. Et Adorno n'est pas plus tendre avec les œuvres de Rachmaninov ou Tchaïkovski qu'avec les rengaines du jazz.

32. Horkheimer, Éclipse de la raison, 112.

33. Ibid., 31.

34. Herbert Marcuse, L'homme unidimensionnel (Paris : Minuit, 1968).

35. Cf. par ex. Theodor W. Adorno et Karl Popper, éd., De Vienne à Francfort. La querelle allemande des sciences sociales (Bruxelles : Éditions Complexe, 1979).

36. Horkheimer, Éclipse de la raison, 32.

37. Alexander Neumann, «Pour une écoute sociologique. Répression, inhibition, prise de parole », Variations. Revue internationale de théorie critique, ${ }^{\mathrm{n}} \mathrm{o} 8$ (2006), §31.

38. Theodor W. Adorno, "Capitalisme tardif ou société industrielle?», in Société: IntégrationDésintégration. Écrits sociologiques (Paris : Payot, 2011), 96.

39. Pour plus d'information sur la situation française à ce propos, on pourra consulter l'ouvrage de Christophe Granger, La destruction de l'université française (Paris : La Fabrique, 2015).

40. Les récents travaux de sciences sociales sur cette "projectification" de la recherche montrent ainsi combien l'activité scientifique est désormais enjointe à «s'aligner sur la dimension managériale $\mathrm{du}$ projet, sur son code sémiotique et narratif», et combien l'assujettissement à la production de livrable voit la recherche tendre à « se conformer à des normes de production qui augmentent le risque de standardisation » qui vont même contre la logique même des appels à projets qui sont censés encourager une recherche originale 
susceptible de «créer de la valeur ajoutée ». Cf. Anne Piponnier, « Le projet dans les pratiques de recherche. Pour un retour réflexif et critique sur nos engagements ", Sciences de la société, ${ }^{\mathrm{n}} \mathrm{O} 93$ (2015), 110-23.

41. On consultera à ce propos les nombreux travaux de l'économie politique de la communication qui montrent combien les mouvements de l'industrie culturelle ont été au cœur d'une redistribution internationale du travail où « la main d'œuvre non qualifiée se concentre dans les pays les plus pauvres, la main d'œuvre plus qualifiée et spécialisée dans l'assemblage se trouve dans les pays semi-périphériques ; quant aux emplois liés à la recherche, au développement et à la planification, ils se concentrent dans les grandes entreprises commerciales des pays riches. » Vincent Mosco, The Political Economy of Communication (London : Sage, 2009), 74. Cf. aussi Christian Fuchs, Digital Labour and Karl Marx (London : Routledge, 2014), particulièrement les chapitres 5 et 6.

42. Theodor W. Adorno, "Engagement », in Notes sur la littérature (Paris: Flammarion, 1984), 286.

43. Jean-Paul Sartre, Qu'est-ce que la littérature? (Paris: Gallimard, 1948).

44. Cf. Gisèle Sapiro, La responsabilité de l'écrivain. Littérature, droit et morale en France (XIXe-XXIe siècle) (Paris : Seuil, 2011).

45. Cf. par ex. Annie Ernaux, L'écriture comme un couteau. Entretien avec Frédéric-Yves Jeannet (Paris : Stock, 2003).

46. D'autant que Sartre n'hésite pas dans son essai à défendre l'importance fonctionnelle des mass media comme outil prolongeant les visées de l'art engagé pour «conquérir le public virtuel».

47. Herbert Marcuse, La dimension esthétique (Paris : Seuil, 1979), 20.

48. Theodor W. Adorno, Théorie esthétique (Paris : Klincksieck, 1995).

49. Marcuse, La dimension esthétique, 12.

50. Adorno, « Engagement », 301.

51. Theodor W. Adorno, "Arnold Schönberg », in Prismes : critique de la culture et société (Paris : Payot, 1986), 153.

52. Theodor W. Adorno, "L'essai comme forme ", in Notes sur la littérature (Paris : Flammarion, 1984), 9.

53. Adorno, « Capitalisme tardif ou société industrielle? », 97.

54. Ibid., 89.

55. Adorno et Popper, De Vienne à Francfort. La querelle allemande des sciences sociales, 62.

56. Theodor W. Adorno, "Un étrange réaliste: Siegfried Kracauer", in Notes sur la littérature (Paris : Flammarion, 1984), 268.

57. Adorno, « L'essai comme forme », 13.

58. Ibid., 24.

59. Max Horkheimer et Theodor W. Adorno, Le laboratoire de la Dialectique de la raison. Discussions, notes et fragments inédits (Paris: Maison des sciences de l'homme, 2013).

60. Adorno et Horkheimer, Towards a new manifesto.

61. Gérard Raulet, «Interdisciplinarité ou essayisme? La "philosophie sociale" de la Dialektik der Aufklärung », in Jenseits instrumenteller Vernunft. Kritische Studien zur « Dialektik der Aufklärung », éd. par Manfred Gangl et Gérard Raulet (Berlin : Peter Lang, 1998).

62. Adorno, "Mode intemporelle ", 16.

63. Nous proposons ici de nommer "désengagement" cette attitude qui consiste à ne se maintenir que dans la production de théorie critique engagée, par opposition de ce que serait un "dégagement» (illusoire), position d'un chercheur qui se penserait comme se tenant objectivement à l'écart de tout «engagement » théorique. Cf. Bensaïd et Corcuff, «Le travail intellectuel au risque de l'engagement ».

64. Alexander Neumann, "Le courant chaud de l'École de Francfort", Variations. Revue internationale de théorie critique, ${ }^{\mathrm{n}} \mathrm{O} 12$ (2008). 
65. «À quoi sert-il de taire la dépression qui le tenait en tenaille durant l'exil américain ? Pourquoi nier sa morgue passagère contre Herbert Marcuse, ou la façon désinvolte dont il traitait son ami Walter Benjamin ? Oui, Adorno était un homme mortel, donc imparfait, il a insulté son concurrent Marcuse d'être un «fasciste entravé » et suggéré à Benjamin de se mettre à l'abri, en adhérant à la ligue des écrivains du régime nazi, mais tout cela n'enlève rien à la pertinence éclatante de ses concepts et arguments. Oui, Adorno a fait intervenir la police pour expulser les trouble-fêtes qui occupaient l'Institut de Francfort en 68. Ses réactions devant le mouvement étudiant contestataire - tantôt désemparé, tantôt engagé - ne font-ils pas partie d'un caractère entier et contrasté ? Les héritiers légaux et symboliques d'Adorno refoulent ces éléments, bien que ceux-ci viennent à leur manière renforcer l'idée du non-identique, selon laquelle l'unité de la théorie et de la pratique est une tâche surhumaine. Les héritiers participent ainsi à la construction d'un mythe, au lieu de rendre sa pensée vivante. », Ibid., § 6 .

66. Cervera-Marzal, Pour un suicide des intellectuels, 23.

67. Ibid., 25.

68. Ibid., 56.

69. Ibid.

70. Ibid., 86.

71. Voir l'article d'Alexander Neumann sur « La bureaucratie liquide » dans ce numéro.

72. Cervera-Marzal, Pour un suicide des intellectuels, 90.

73. Adorno rappelle d'ailleurs à ce titre combien le teamwork en recherche social est une forme de la réification dans le monde de la recherche : « au sein du teamwork, [les êtres humains] ne sont fondamentalement rien d'autre que des fonctions partielles plus imparfaites d'un écanisme dont la finalité n'entre pour rien dans leur travail lui-même et, pour cette raison, ne les amène pas du tout à une véritable solidarité. », Theodor W. Adorno, "Le teamwork en recherche sociale ", in Le conflit des sociologies. Théorie critique et sciences sociales (Paris: Payot, 2016), 310. L'importance du mot-clef «interdisciplinarité » dans l'attribution de financements à la recherche par les organismes nationaux ou européens dédiés laisse ainsi bien entendre que le projet d'une telle «ouverture " au-delà des spécialités disciplinaires n'est en rien une remise en cause de la balkanisation des savoirs scientifiques en elle-même mais plutôt sa légitimation par la croyance que certaines thématiques ou objets contemporains nécessiteraient justement l'apport hyperspécialisé de plusieurs chercheurs venant de domaines divers.

74. Cervera-Marzal, Pour un suicide des intellectuels, 138.

75. Cf. par ex. Bensaïd et Corcuff, « Le travail intellectuel au risque de l'engagement ».

76. Perry Anderson, Sur le marxisme occidental (Paris : Maspéro, 1977).

77. John Holloway, Crack capitalism. 33 thèses contre le capital (Paris : Libertalia, 2012).

78. Ibid., 35.

79. Ibid., 36-37.

80. Ibid., 88 .

81. Ibid., 83.

82. Ibid., 415.

83. Ibid., 407.

84. Ibid., 413.

85. Ibid., 424.

86. John Holloway, Crack capitalism. 33 thèses contre le capital, 414.

87. Adorno et Horkheimer, Towards a new manifesto, 102. 
INDEX

Mots-clés : travail intellectuel, Théorie critique, Adorno Theodor, Horkheimer Max, industrie culturelle, art, engagement

\section{AUTEUR}

\section{CHRISTOPHE MAGIS}

Maître de Conférences en Sciences de l'information et de la communication, Université Paris 8 Cemti 